\title{
Thermal tuning of mid-infrared plasmonic antenna arrays using a phase change material
}

\section{Citation}

Kats, Mikhail A., Romain Blanchard, Patrice Genevet, Zheng Yang, M. Mumtaz Qazilbash, D. N. Basov, Shriram Ramanathan, and Federico Capasso. 2013. "Thermal Tuning of Mid-Infrared Plasmonic Antenna Arrays Using a Phase Change Material." Optics Letters 38 (3): 368. https:// doi.org/10.1364/ol.38.000368.

\section{Permanent link}

http://nrs.harvard.edu/urn-3:HUL.InstRepos:41371344

\section{Terms of Use}

This article was downloaded from Harvard University's DASH repository, WARNING: This file should NOT have been available for downloading from Harvard University's DASH repository.

\section{Share Your Story}

The Harvard community has made this article openly available.

Please share how this access benefits you. Submit a story.

Accessibility 


\title{
Thermal tuning of mid-infrared plasmonic antenna arrays using a phase change material
}

\author{
Mikhail A. Kats, ${ }^{1}$ Romain Blanchard, ${ }^{1}$ Patrice Genevet, ${ }^{1}$ Zheng Yang, ${ }^{1}$ M. Mumtaz Qazilbash, ${ }^{2,3}$ \\ D. N. Basov, ${ }^{2}$ Shriram Ramanathan, ${ }^{1}$ and Federico Capasso ${ }^{1, *}$ \\ ${ }^{1}$ School of Engineering and Applied Sciences, Harvard University, Cambridge, Massachusetts 02138, USA \\ ${ }^{2}$ Department of Physics, University of California-San Diego, La Jolla, California 92093, USA \\ ${ }^{3}$ Department of Physics, The College of William \& Mary, Williamsburg, Virginia 23187, USA \\ ${ }^{*}$ Corresponding author: capasso@seas.harvard.edu
}

Received November 16, 2012; revised December 20, 2012; accepted December 30, 2012; posted January 2, 2013 (Doc. ID 179906); published January 29, 2013

\begin{abstract}
We demonstrate that the resonances of infrared plasmonic antennas can be tuned or switched on/off by taking advantage of the thermally driven insulator-to-metal phase transition in vanadium dioxide $\left(\mathrm{VO}_{2}\right)$. Y-shaped antennas were fabricated on a $180 \mathrm{~nm}$ film of $\mathrm{VO}_{2}$ deposited on a sapphire substrate, and their resonances were shown to depend on the temperature of the $\mathrm{VO}_{2}$ film in proximity of its phase transition, in good agreement with full-wave simulations. We achieved tunability of the resonance wavelength of approximately $10 \% \quad(>1 \mu \mathrm{m}$ at $\lambda \sim 10 \mu \mathrm{m})$. (C) 2013 Optical Society of America
\end{abstract}

OCIS codes: $250.5403,240.6680,130.3060,130.4815$.

Optical antennas have in recent years become a backbone of optics at subwavelength scales. Due to localized surface plasmon resonances arising from the collective motion of free electrons in metals, optical antennas have become useful for sensing, photodetection, heat transfer, and emission enhancement schemes [1-5]. More recently, optical antennas have also become relevant for the manipulation of the amplitude, phase, and polarization of light [6-10].

To date, most of the applications that utilize optical antennas have involved static, nonreconfigurable designs. These applications all stand to benefit from dynamic tunability. To this end, a number of approaches have been investigated, including antenna loading with liquid crystals [11] and photoconductive materials [12], tuning the gap of dimer antennas with scanning probes [13] or elastomeric substrates $[14,15]$, and modification of the local environment with active polymer coatings [16].

In this Letter, we demonstrate that resonances of midinfrared (IR) plasmonic antennas can be tuned and switched on/off by thermal control of phase coexistence in a vanadium dioxide $\left(\mathrm{VO}_{2}\right)$ substrate. A similar approach has been previously explored for tuning of the effective refractive index of metamaterials in the terahertz (THz) [17-19] and near-IR [20], tuning nanoparticle scattering spectra in the visible [21-23], and control of transmission through apertures in the $\mathrm{THz}$ [24]. We utilized a normalization procedure that helps isolate the antenna resonances from the optical response of the unpatterned underlying substrate in proximity to the $\mathrm{VO}_{2}$ phase transition [25]. To validate our results, we incorporated the literature complex refractive indices of $\mathrm{VO}_{2}$ throughout its phase transition into full-wave numerical simulations and observed qualitative agreement between the simulated and experimental reflectivity spectra.

$\mathrm{VO}_{2}$ undergoes a thermally driven insulator-to-metal transition (IMT) above $\sim 67^{\circ} \mathrm{C}$, which takes the material from an insulating state to a metallic state. In recent years this phase transition has been explored for the development of novel electronic switches and memories [26]. In the insulating phase, $\mathrm{VO}_{2}$ has a bandgap corresponding to $\lambda=1.8-2.5 \mu \mathrm{m}$, depending on the growth conditions (e.g., [27]), and in the metallic phase it has Drude-like optical conductivity at IR frequencies [28]. This large change in optical properties makes $\mathrm{VO}_{2}$ films useful for optical switching applications [29,30]. As the IMT occurs, the bandgap collapses and nanoscale islands of the metallic phase begin to form in the surrounding insulating $\mathrm{VO}_{2}$, which then grow and connect in a percolative process $[27,31]$. This nanoscale phase coexistence makes intermediate-state $\mathrm{VO}_{2}$ an effective optical medium with tunable dispersion and losses [25].

A plasmonic antenna can be made tunable by incorporating a material such as $\mathrm{VO}_{2}$ into its design; here, we focus on Y-shaped antennas that can be used as phase elements and form birefringent metasurfaces [10]. We fabricated a square array of gold $(\mathrm{Au}) \mathrm{Y}$-shaped antennas with a period of $3 \mu \mathrm{m}$ on a thick, single-side-polished cplane sapphire substrate coated with $\sim 180 \mathrm{~nm}$ of singlecrystalline $\mathrm{VO}_{2}$. The antenna spacing is large to minimize near-field coupling between neighboring antennas. The oxide film was deposited using magnetron sputtering from a $\mathrm{V}_{2} \mathrm{O}_{5}$ target at $550^{\circ} \mathrm{C}$ under 10 mTorr pressure with $100 \mathrm{sccm}$ Ar gas flow at a power of $120 \mathrm{~W}$ to obtain phase pure $\mathrm{VO}_{2}$. The antennas were defined using electronbeam lithography and deposition of $5 \mathrm{~nm}$ of $\mathrm{Ti}$ and $40 \mathrm{~nm}$ of $\mathrm{Au}$, and lift-off. A scanning electron microscope (SEM) image of the structures is shown in Fig. 1(a); the vertical and horizontal arms are $\sim 1.05 \mu \mathrm{m}$ long and the diagonal arm is $\sim 0.75 \mu \mathrm{m}$ long, all with a width of $\sim 160 \mathrm{~nm}$. The roughness of the $\mathrm{VO}_{2}$ film is visible in the SEM image and was transferred to the metallic antennas.

An antenna array situated on a dielectric substrate can be viewed as a frequency selective surface and is expected to have a peak in its reflectivity spectrum when on resonance [32]. We collected temperature-dependent reflectivity spectra by using a Fourier transform infrared (FTIR) spectrometer (Bruker Vertex 70), connected to a mid-IR microscope (Bruker Hyperion 2000) equipped with a temperature-controlled stage (Bruker A599). A schematic of the setup is shown in Fig. 1(b). A Cassegrain objective $(15 \times, \mathrm{NA}=0.4)$ was used to focus a polarized 

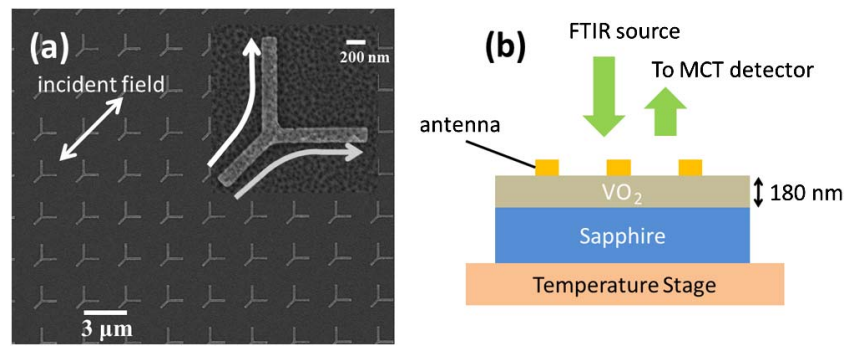

Fig. 1. (Color online) (a) SEM image of the fabricated sample comprising an array of gold antennas on a $\sim 180 \mu \mathrm{m} \mathrm{VO}_{2}$ film deposited on a sapphire wafer. Inset: zoomed-in image of a single Y-shaped antenna with arrows indicating the flow of the current. (b) Schematic of the reflectivity measurement. Light from an FTIR is incident on the temperature-controlled sample, and the reflection is sent to a detector.

beam from a Globar source onto the sample, with the reflected light collected by the same objective and sent to a mercury-cadmium-telluride (MCT) detector. Nearly all of the light that is not reflected is absorbed within the antennas, the $\mathrm{VO}_{2}$ film, and the underlying sapphire as it is not transparent for $\lambda>5 \mu \mathrm{m}$. At each temperature, the reflectivity spectra were normalized to (i.e., divided by) the reflectivity of the bare $\mathrm{VO}_{2}$ /sapphire substrate at that same temperature. Such normalization helps isolate the reflectivity feature due to the antennas at each temperature from the nontrivial reflectivity of the underlying $\mathrm{VO}_{2}$ film on sapphire [25].

The normalized reflectivity is shown in Fig. 2(a), with the incident polarization along the symmetry axis of the antennas as indicated with the double-sided arrow in Fig. 1(a); this ensures that no polarization conversion takes place at the antenna array [10]. When the $\mathrm{VO}_{2}$ is in the dielectric state (e.g., $T=28^{\circ} \mathrm{C}$ ), the resonances of the antennas in the array lead to a peak in reflectivity at around $\lambda=9.3 \mu \mathrm{m}$. As the temperature increases to $T=69^{\circ} \mathrm{C}$, this peak is redshifted to $\lambda=10.5 \mu \mathrm{m}$, corresponding to a greater than $10 \%$ shift in the resonance frequency. This shift coincides with a decrease in the peak reflectivity and increase in the linewidth. The wavelength of the reflectivity peak and its value are plotted in Fig. 2(b) as a function of temperature when heating (solid curves) and then cooling back down (dashed curves). There is hysteresis in this plot that is inherent to the
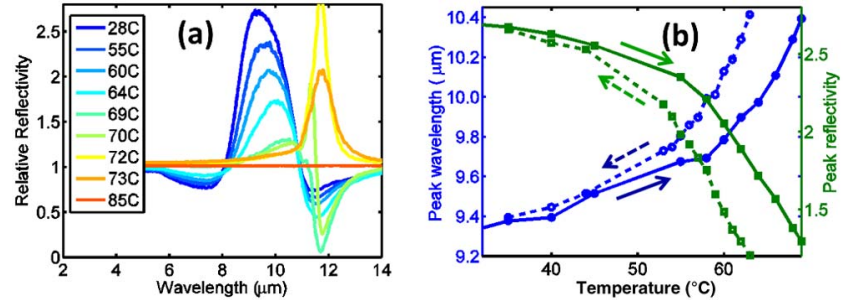

Fig. 2. (Color online) (a) Reflectivity spectra of the antenna array for increasing temperatures, showing the evolution of the antenna resonance through the $\mathrm{VO}_{2}$ phase transition. At each temperature, the spectrum was normalized to reflectivity of the substrate without the antennas. (b) Wavelength at which the reflectivity peaks [blue (lower left to upper right)] and the normalized peak reflectivity value [green (upper left to lower right)] as a function of temperature during heating (solid curves) and cooling (dashed curves).
$\mathrm{VO}_{2}$ phase transition [26], though the full hysteresis loop is not visible in Fig. $2(\mathrm{~b})$ because we truncated the temperatures at $69^{\circ} \mathrm{C}$ for heating and $63^{\circ} \mathrm{C}$ for cooling, respectively; beyond these temperatures the reflectivity peak disappears.

The key features of Fig. 2(a) are reproduced by threedimensional finite-difference time-domain (FDTD) simulations (Lumerical Solutions) in Fig 3(a). Periodic boundary conditions were used to capture the effect of the array. The IR complex refractive index data for sapphire, $\mathrm{Ti}$, and $\mathrm{Au}$ was interpolated from literature data [33], while the $\mathrm{VO}_{2}$ refractive indices throughout its phase transition were taken from ellipsometry measurements in [28]. The differences between the experimental results of Fig. 2(a) and the calculations of Fig. 3(a) are primarily due to the differing growth conditions and thicknesses between our $\mathrm{VO}_{2}$ sample and the one measured in [28], which lead to a different temperature-dependent complex refractive index, as well as the surface roughness that is not accounted for in the simulations.

This shift in the resonance occurs because the refractive index of the underlying $\mathrm{VO}_{2}$ changes as a function of temperature. At the onset of the transition, the real part of the complex refractive index $n$ increases at mid-IR wavelengths [curves for 9.3 and $10.5 \mu \mathrm{m}$ are shown in blue (upper on the left, lower on the right) in Fig. 3(b)]. This increase in the substrate index is strongly felt by the antenna, which acts as a dispersive Fabry-Perot resonator for surface plasmons with the effective index of the mode dependent on the index of the substrate $[34,35]$. In the present demonstration, we are exciting one of the two plasmonic eigenmodes of the Y-shaped antenna [10]; the other eigenmode feels the index change of the substrate in the same way. Through the course of the $\mathrm{VO}_{2}$ phase transition the antenna resonance is also impacted by the increase of losses [the values of the imaginary part $k$ of the complex refractive index of $\mathrm{VO}_{2}$ are shown in black (lower on the left, upper on the right) in Fig. 3(b)], which increase as the fraction of the metallic-phase $\mathrm{VO}_{2}$ increases, resulting in a lower-amplitude reflectivity maximum. As the $\mathrm{VO}_{2}$ film transitions into its hightemperature metallic state (where $k>n$ ), the antennas become "shorted" and can no longer be viewed as isolated resonant elements, so the reflectivity maximum disappears [flat orange curves in Figs. 2(a) and 3(a)]. The peak in normalized reflectivity in Figs. 2(a) and 3(a) at 11-12 $\mu \mathrm{m}$ that appears at temperatures close to the
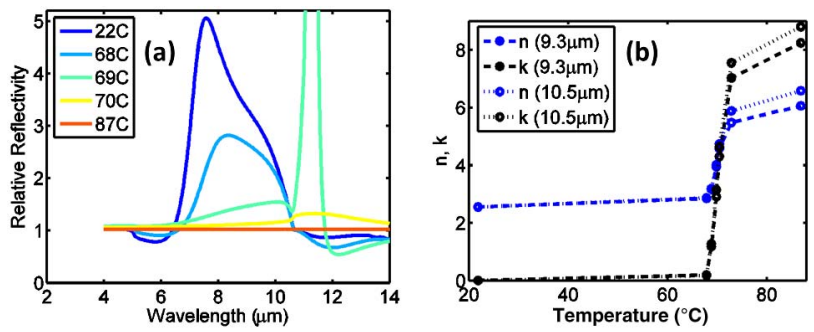

Fig. 3. (Color online) (a) Calculated normalized reflectivity spectra corresponding to the experimental data in Fig. 2(a) using the FDTD method, incorporating literature values for the complex refractive indices of gold, $\mathrm{VO}_{2}$, and sapphire. (b) Real $(n)$ and imaginary $(k)$ parts of the refractive index of $\mathrm{VO}_{2}$ at wavelengths of 9.3 and $10.5 \mu \mathrm{m}$. 
critical IMT temperature is a result of a "division by zero": at this wavelength and temperature the reflectivity of the underlying $\mathrm{VO}_{2}$ /sapphire substrate is $\sim 0$ as a result of critical coupling to an ultrathin cavity resonance [25], causing a divergence in the normalized curves. The dip at this same wavelength but at a slightly lower temperature [Fig. 2(a)] is an indication that the critical coupling condition is reached for a lower temperature in the presence of antennas due to enhanced absorption in the $\mathrm{VO}_{2}$.

The manipulation of optical antenna resonances using phase change materials such as $\mathrm{VO}_{2}$ is promising for dynamic optical devices. In this Letter, we showed that the resonances of plasmonic elements can be tuned or turned off by utilizing the large change of the optical properties of the underlying $\mathrm{VO}_{2}$ substrate in proximity to its phase transition. While we demonstrated thermal tuning of an entire antenna array, DC and AC fields can be applied to individual antennas or even individual sections of antennas on isolated patches of $\mathrm{VO}_{2}$, creating a tunable inhomogeneous distribution of amplitude and phase responses within an array of antennas. Such a demonstration would be of interest to realizing antenna-based spatial light modulators capable of addressing amplitude and phase of infrared and THz light, active and passive thermal labels and emitters, displays, and related dynamically reconfigurable devices.

We thank J. Lin, N. Yu, D. Sharma, and Y. Zhao for helpful discussions. The fabrication was performed at the Harvard CNS. FC acknowledges support from the AFOSR under grant number FA9550-12-1-0289. ZY and SR acknowledge ONR for financial support. M. Kats is supported by the NSF Graduate Research Fellowship.

\section{References}

1. K. B. Crozier, A. Sundaramurthy, G. S. Kino, and C. F. Quate, J. Appl. Phys. 94, 4632 (2003).

2. P. Muhlschlegel, H.-J. Eisler, O. J. F. Martin, B. Hecht, and D. W. Pohl, Science 308, 1607 (2005).

3. R. Adato, A. A. Yanik, J. J. Amsden, D. L. Kaplan, F. G. Omenetto, M. K. Hong, S. Erramilli, and H. Altug, Proc. Natl. Acad. Sci. USA 106, 19227 (2009).

4. L. Novotny and N. van Hulst, Nat. Photonics 5, 83 (2011).

5. E. Cubukcu, N. Yu, E. J. Smythe, L. Diehl, K. B. Crozier, and F. Capasso, IEEE J. Sel. Top. Quantum Electron. 14, 1448 (2008).

6. N. Yu, P. Genevet, M. A. Kats, F. Aieta, J. P. Tetienne, F. Capasso, and Z. Gaburro, Science 334, 333 (2011).

7. Y.-J. Tsai, S. Larouche, T. Tyler, G. Lipworth, N. M. Jokerst, and D. R. Smith, Opt. Express 19, 24411 (2011).

8. M. A. Kats, N. Yu, P. Genevet, Z. Gaburro, and F. Capasso, Opt. Express 19, 21749 (2011).

9. Y. Zhao and A. Alu, Phys. Rev. B 84, 205428 (2011).

10. M. A. Kats, P. Genevet, G. Aoust, N. Yu, R. Blanchard, F. Aieta, Z. Gaburro, and F. Capasso, Proc. Natl. Acad. Sci. USA 109, 12364 (2012).

11. J. Berthelot, A. Bouhelier, C. Huang, J. Margueritat, G. Colas-des-Francs, E. Finot, J.-C. Weeber, A. Dereux, S. Kostcheev, H. ibn El Ahrach, A.-L. Baudrion, J. Plain,
R. Bachelot, P. Royer, and G. P. Wiederrecht, Nano Lett. 9, 11 (2009).

12. N. Large, M. Abb, J. Aizpurua, and O. L. Muskens, Nano Lett. 10, 1741 (2010).

13. P. Olk, J. Renger, M. T. Wenzel, and L. M. Eng, Nano Lett. 8, 4 (2008).

14. F. Huang and J. J. Baumberg, Nano Lett. 10, 5 (2010).

15. I. M. Pryce, K. Aydin, Y. A. Kelaita, R. M. Briggs, and H. A. Atwater, Nano Lett. 10, 4222 (2010).

16. H. Gehan, C. Mangeney, J. Aubard, G. Levi, A. Hohenau, J. R. Krenn, E. Lacaze, and N. Felidj, J. Phys. Chem. Lett. 2, 926 (2011).

17. T. Driscoll, S. Palit, M. M. Qazilbash, M. Brehm, F. Keilmann, B.-G. Chae, S.-J. Yun, H.-T. Kim, S. Y. Cho, N. M. Jokerst, D. R. Smith, and D. N. Basov, Appl. Phys. Lett. 93, 024101 (2008).

18. M. D. Goldflam, T. Driscoll, B. Chapler, O. Khatib, N. M. Jokerst, S. Palit, D. R. Smith, B.-J. Kim, G. Seo, H.-T. Kim, M. D. Ventra, and D. N. Basov, Appl. Phys. Lett. 99, 044103 (2011).

19. M. Liu, H. Y. Hwang, H. Tao, A. C. Strikwerda, K. Fan, G. R. Keiser, A. J. Sternbach, K. G. West, S. Kittiwatanakul, J. Lu, S. A. Wolf, F. G. Omenetto, X. Zhang, K. A. Nelson, and R. D. Averitt, Nature 487, 345 (2012).

20. M. J. Dicken, K. Aydin, I. M. Pryce, L. A. Sweatlock, E. M. Boyd, S. Walavalker, J. Ma, and H. A. Atwater, Opt. Express 17, 18330 (2009).

21. J. Y. Suh, E. U. Donev, D. W. Ferrara, K. A. Ketz, and L. C. Feldman, J. Opt. A 10, 055202 (2008).

22. D. Y. Lei, K. Appavoo, Y. Sonnefraud, R. F. Haglund, and S. A. Maier, Opt. Lett. 35, 23 (2010).

23. D. W. Ferrara, E. R. MacQuarrie, J. Nag, A. B. Kaye, and R. F. Haglund, Appl. Phys. Lett. 98, 241112 (2011).

24. M. Seo, J. Kyoung, H. Park, S. Koo, H. Kim, H. Bernien, B. J. Kim, J. H. Choe, Y. H. Ahn, H.-T. Kim, N. Park, Q-H. Park, K. Ahn, and D. Kim, Nano Lett. 10, 2064 (2010).

25. M. A. Kats, D. Sharma, J. Lin, P. Genevet, R. Blanchard, Z. Yang, M. M. Qazilbash, D. Basov, S. Ramanathan, and F. Capasso, Appl. Phys. Lett. 101, 221101 (2012).

26. Z. Yang, C. Ko, and S. Ramanathan, Annu. Rev. Mater. Res. 41, 337 (2011).

27. W.-T. Liu, J. Cao, W. Fan, Z. Hao, M. C. Martin, Y. R. Shen, J. Wu, and F. Wang, Nano Lett. 11, 466 (2011).

28. M. M. Qazilbash, M. Brehm, B.-G. Chae, P.-C. Ho, G. O. Andreev, B.-J. Kim, S. J. Yun, A. V. Balatsky, M. B. Maple, F. Keilmann, H.-T. Kim, and D. N. Basov, Science 318, 1750 (2007).

29. L. A. Gea and L. A. Boatner, Appl. Phys. Lett. 68, 3081 (1996).

30. D. Ruzmetov, K. T. Zawilski, S. D. Senanayake, V. Narayanamurti, and S. Ramanathan, J. Phys. 20, 465205 (2008).

31. H. S. Choi, J. S. Ahn, J. H. Jung, T. W. Noh, and D. H. Kim, Phys. Rev. B 54, 4621 (1996).

32. B. A. Munk, Frequency Selective Surfaces: Theory and Design (Wiley-Interscience, 2000).

33. E. D. Palik, Handbook of Optical Constants of Solids II (Elsevier, 1998).

34. I. Puscasu, D. Spencer, and G. D. Boreman, Appl. Opt. 39, 1570 (2000).

35. E. Cubukcu and F. Capasso, Appl. Phys. Lett 95, 201101 (2009). 